\title{
Article
}

\section{Differences in the kinetics and kinematics of supported and un-supported landings of the rugby union lineout}

Sinclair, Jonathan Kenneth, Smith, Adam, Taylor, Paul John and Hobbs, Sarah Jane

Available at http://clok.uclan.ac.uk/16484/

Sinclair, Jonathan Kenneth ORCID: 0000-0002-2231-3732, Smith, Adam, Taylor, Paul John ORCID: 0000-0002-9999-8397 and Hobbs, Sarah Jane ORCID: 0000-0002-1552-8647 (2017) Differences in the kinetics and kinematics of supported and un-supported landings of the rugby union lineout. Comparative Exercise Physiology, 13 (1). pp. 1-6. ISSN 1755-2540

It is advisable to refer to the publisher's version if you intend to cite from the work. http://dx.doi.org/10.3920/CEP160029

For more information about UCLan's research in this area go to http://www.uclan.ac.uk/researchgroups/ and search for <name of research Group>.

For information about Research generally at UCLan please go to http://www.uclan.ac.uk/research/

All outputs in CLoK are protected by Intellectual Property Rights law, including Copyright law. Copyright, IPR and Moral Rights for the works on this site are retained by the individual authors and/or other copyright owners. Terms and conditions for use of this material are defined in the policies page. 
1 Differences in the kinetics and kinematics of supported and un-supported landings of

\section{Corresponding author contact details:}

9 Jonathan Sinclair

10 Centre for Applied Sport and Exercise Sciences

11 School of Sport \& Wellbeing

12 College of Health \& Wellbeing

13 University of Central Lancashire

14 Preston

15 Lancashire

16 PR1 2HE

17 E-mail: jksinclair@uclan.ac.uk

18

19 Keywords: Rugby, lineout, biomechanics.

20

\section{Abstract}

22 The aim of the current investigation was to comparatively examine the kinetics and 23 kinematics of supported and un-supported landings during the rugby union line out. Eleven Lancashire, UK. 
male line-out jumpers were tested under two conditions, 'supported' in which the lifters maintained supportive contact with the jumper until the jumpers' feet touched the floor and 'un-supported' in which the lifters released the jumper once they had caught the ball. Kinematics were examined using an eight camera motion capture system and kinetics using a force platform. Differences between conditions were examined using paired t-tests. The findings showed the instantaneous loading rate (supported $=212.9 \pm 102.5 \mathrm{BW} / \mathrm{s} \&$ unsupported $=449.0 \pm 142.4 \mathrm{BW} / \mathrm{s}$ ) and vertical velocity (supported $=2.7 \pm 0.4 \mathrm{~m} / \mathrm{s} \&$ unsupported $=4.0 \pm 0.4 \mathrm{~m} / \mathrm{s}$ ) at foot contact were significantly larger in the un-supported condition. The findings from the current investigation indicate that if the line-out jumper is un-supported by the lifters in returning to the ground then their risk from injury is likely to be greater. Therefore, given the number of line-outs that are conducted per game it is recommended that this law be clarified to also specify supported lowering of the jumper at all levels of play.

\section{Introduction}

In rugby union the lineout is a fundamental mechanism for restarting the game when the ball has left the field (Trewartha et al., 2008). The lineout is accomplished when the thrower throws the ball infield towards the two opposing units of jumpers and lifters whose aim it is to retain/ regain possession of the ball (Sayers, 2011). The lineout is a key attacking platform in rugby union that provides a mechanism for scoring opportunities (Trewartha et al., 2008). In professional rugby match play, the team who is in possession (i.e. the team that initiate the infield throw) will subsequently acquire possession of the ball in around $80 \%$ instances and $26 \%$ of all tries are attained after securing possession of the ball directly from a lineout (Trewartha et al., 2008). 
In professional level rugby union matches there are approximately 34 lineouts in each game, (IRB, 2007). The ball must be thrown directly down the middle of the two opposing teams (separated by a gap of $1 \mathrm{~m}$ ), thus teams must utilize a range of mechanisms in an attempt to secure possession. The principal manner by which this is achieved is by having the lifters hoist the jumper as high as possible allowing them to catch the ball prior to the opposition

54 (Croft et al., 2011). Due to this the majority of lineout throws are now caught at a height of around $3.5 \mathrm{~m}$ (Sayers, 2011). The mass of the jumper is distributed equally between lifters at the start of the motion; however this is then transferred towards the rear lifter towards the end of the lineout (Sayers, 2011).

Once the ball has been caught and the lifters from both teams release the jumper resulting in a landing for the jumper. As such whilst each of the distinct positions in the lineout places different stresses on the body, given the height at which they are landing from it is likely that the jumpers are at greatest risk from musculoskeletal injury during the lineout. This notion is supported by the observations of Bathgate et al., (2002) who demonstrated that second row forwards are at the highest risk from injury in relation to all other players. Similarly, Brooks \& Kemp, (2011) showed that firstly that second row forwards were at greater risk from injury at the Achilles tendon, ankle collateral ligament and knee anterior/ medial collateral ligaments in relation to other forwards and secondly that a higher proportion of these injuries were sustained as a function of the lineout in relation to other forwards. The World-Rugby Law 19.10 (g) indicates that ' players who support a jumping team-mate must lower that player to the ground as soon as the ball has been won by a player of either team” (IRB, 2005). This rule is somewhat ambiguous in that it does not stipulate that 
supported lowering of the jumper by the lifters is a specific requirement. Rather it mandates

74

\section{Participants}

94 Eleven male rugby union players volunteered to take part in this investigation. Each player

that the lifters must not continue to support the jumper in the air once the ball is secured by either the attacking or defensive side. Therefore, in their haste to make it quickly to the next play, the jumper rotating $90^{\circ}$ in order to set-up a driving maul from an attacking lineout, interference from the opposing jumpers challenging for the ball or competition from opposing forwards necessitating the rapid establishment of an attacking/ defensive maul; lifters may neglect or are unable to support the jumper appropriately in returning to the ground (Patton et al., 2006).

Despite the importance of the line-out to success in modern rugby union there is currently a paucity of published biomechanical information regarding the line-out and the majority has concerned the mechanics of the thrower (Sayers, (2005; Trewartha et al., 2008). However, whilst there is some information in scientific literature concerning the biomechanics of the thrower and the accuracy of the throw, there is currently no information regarding the mechanics of the jumper. Therefore, the aim of the current investigation was to examine the kinetics and kinematics of supported and un-supported landings during the rugby union line out. The current investigation may give important information to officials regarding the interpretation and clarity of law World-Rugby Law 19.10 (g).

\section{Methods}

had a minimum of 2 years of lineout jumping experience and played competitive rugby union 
at university first team level. All participants were free from musculoskeletal pathology at the

97 time of data collection and provided written informed consent in accordance with the principles outlined in the Declaration of Helsinki. The mean characteristics of the participants were: age $22 \pm 4$ years, height $1.9 \pm 0.1 \mathrm{~m}$ and body mass $93 \pm 6 \mathrm{~kg}$. The procedure utilized

100 for this investigation was approved by the University of Central Lancashire, Science,

101 Technology, Engineering and Mathematics, ethical committee.

102

103

\section{Procedure}

104

The test protocol required jumpers to catch 10 throws ( 5 supported and 5 un-supported) from a single thrower with 5 years of lineout throwing experience who competed at university first team level. World-Rugby Law mandates that front of the lineout must be at least $5 \mathrm{~m}$ infield,

107 therefore in order to simulate a throw to a jumper at the front of the lineout, a linear distance

108 of $6 \mathrm{~m}$ was chosen. The jumpers all wore taped jumping supports on their thighs and were supported by the same two lifters throughout, who had a minimum of 5 years of lineout lifting experience and who also were competitive at university first team level. In the

111 supported condition the lifters were instructed to maintain supportive contact with the jumper

112 until the point at which the jumpers' feet touched the floor, whereas in the un-supported

113 condition the lifters were required to release the jumper once they had caught the ball. The

114 lifters and jumpers were positioned so that the jumpers dominant foot landed on an embedded

115 piezoelectric force platform (Kistler, Kistler Instruments Ltd., Alton, Hampshire). To prevent

116 any order effects, the supported and un-supported conditions were presented in a

117 counterbalanced manner whereby five participants performed their supported trials first

118 followed by the un-supported trials and vice versa. Participants (lifters and jumpers) were required to undergo a traditional warm-up procedure and several minutes of practice lineout 
drills prior to the commencement of data collection. The landing movement was defined as

121 the duration from foot contact (defined as $>20 \mathrm{~N}$ of vertical force applied to the force 122 platform) to maximum knee flexion.

124 Kinematics and ground reaction forces data were synchronously collected via an analogue

125 board. Kinematic data was captured at $250 \mathrm{~Hz}$ via an eight camera motion analysis system

126 (Qualisys Medical AB, Goteburg, Sweden). Dynamic calibration of the motion capture

127 system was performed before each data collection session. Lower extremity segments were

128 modelled in 6 degrees of freedom using the calibrated anatomical systems technique

129 (Cappozzo et al., 1995). To define the segment co-ordinate axes of the foot, shank and thigh, 130 retroreflective markers were placed bilaterally onto 1st metatarsal, 5th metatarsal, calcaneus, medial and lateral malleoli, medial and lateral epicondyles of the femur. To define the pelvis segment further markers were posited onto the anterior (ASIS) and posterior (PSIS) superior iliac spines. Carbon fiber tracking clusters were positioned onto the shank and thigh segments. The foot was tracked using the 1st metatarsal, 5th metatarsal and calcaneus markers and the pelvis using the ASIS and PSIS markers. The centres of the ankle and knee joints were delineated as the mid-point between the malleoli and femoral epicondyle markers

137 (Sinclair et al., 2015; Graydon et al., 2015), whereas the hip joint centre was obtained using 138 the positions of the ASIS markers (Sinclair et al., 2014). Static calibration trials were 139 obtained allowing for the anatomical markers to be referenced in relation to the tracking 140 markers/ clusters. The Z (transverse) axis was oriented vertically from the distal segment end 141 to the proximal segment end. The Y (coronal) axis was oriented in the segment from posterior 142 to anterior. Finally, the X (sagittal) axis orientation was determined using the right hand rule 143 and was oriented from medial to lateral. 
145 Data processing

146 Lineout trials from both supported and un-supported conditions were processed in Qualisys

147 Track Manager and then exported as C3D files. Kinematic parameters were quantified using

148 Visual 3-D (C-Motion Inc, Gaithersburg, USA) after marker data was smoothed using a low-

149 pass Butterworth 4th order zero-lag filter at a cut off frequency of $15 \mathrm{~Hz}$. Kinematics of the

150 hip, knee and ankle were quantified using an XYZ cardan sequence of rotations (where $\mathrm{X}$ is

151 flexion-extension; $\mathrm{Y}$ is ab-adduction and is $\mathrm{Z}$ is internal-external rotation). All data were

152 normalized to $100 \%$ of the landing phase then processed trials were averaged. Sagittal plane

153 kinematic measures from the hip, knee and ankle which were extracted for statistical analysis

154 were 1) angle at foot contact 2) angle at landing termination, 3) peak angle during landing, 4)

155 angular range of motion (ROM) from footstrike to landing termination, and 5) relative ROM

156 from foot contact to peak angle.

157

158 From the force platform instantaneous loading rate was calculated as the maximum increase

159 in vertical force between adjacent data points (Sinclair et al., 2013). The instantaneous

160 loading rate was normalized by dividing the values by each participant's body weight

161 (BW/s). In addition limb stiffness was quantified using a mathematical spring-mass model

162 Blickman, (1989). Limb stiffness was calculated by dividing the peak vertical GRF by the

163 amount of limb compression (Farley \& Morgenroth, 1999). Limb stiffness was normalized to

164 by dividing by participant’s bodyweight (BW/m).

165

166

Statistical analyses 
167 Descriptive statistics (means and standard deviations) were obtained for each line-out

168 condition. Shapiro-Wilk tests were used to screen the data for normality. Differences in

169 kinetic and kinematic parameters were examined using paired t-tests. Statistical significance

170 was accepted at the $\mathrm{P} \leq 0.05$ level. All statistical actions were conducted using SPSS v22.0

171 (SPSS Inc, Chicago, USA).

172

173 Results

$174 \quad$ Kinetics

175 The kinetic analysis showed that instantaneous loading rate was significantly ( $\mathrm{t}=5.54$,

$176 \mathrm{P}<0.05)$ larger in the un-supported $(449.0 \pm 142.4 \mathrm{BW} / \mathrm{s})$ condition in relation to supported

177 (212.9 $\pm 102.5 \mathrm{BW} / \mathrm{s})$. In addition it was revealed that limb stiffness was significantly

$178(\mathrm{t}=5.03, \mathrm{P}<0.05)$ greater in the supported $(8.5 \pm 2.6 \mathrm{BW} / \mathrm{m})$ condition compared to un-

179 supported $(5.5 \pm 2.0 \mathrm{BW} / \mathrm{m})$.

180

$181 \quad$ Kinematics

182 The kinematic analysis showed that vertical velocity at foot contact was significantly

$183(\mathrm{t}=10.02, \mathrm{P}<0.05)$ greater in the un-supported $(4.0 \pm 0.3 \mathrm{~m} / \mathrm{s})$ condition compared to

184 supported $(2.7 \pm 0.4 \mathrm{~m} / \mathrm{s})$.

185

186 Hip

187

@@@Table1 near here@@@ 
189 For the angle at landing termination the hip was shown to be flexed to a significantly ( $\mathrm{t}=6.15$,

$190 \mathrm{P}<0.05)$ greater extent in the un-supported condition. In addition, peak hip flexion was found 191 to the significantly $(\mathrm{t}=6.02, \mathrm{P}<0.05)$ greater in the un-supported condition. Finally, both ROM $192(\mathrm{t}=10.04, \mathrm{P}<0.05)$ and relative $\mathrm{ROM}(\mathrm{t}=9.59, \mathrm{P}<0.05)$ were shown to be significantly larger 193 in the un-supported condition.

197 For the angle at landing termination landing termination the knee was shown to be flexed to a

198 significantly $(\mathrm{t}=6.89, \mathrm{P}<0.05)$ greater extent in the un-supported condition. In addition, peak 199 knee flexion was found to the significantly $(\mathrm{t}=6.75, \mathrm{P}<0.05)$ greater in the un-supported 200 condition. Finally, both ROM ( $t=5.74, \mathrm{P}<0.05)$ and relative ROM $(\mathrm{t}=5.67, \mathrm{P}<0.05)$ were 201 shown to be significantly larger in the un-supported condition.

Ankle

205 In addition, peak dorsiflexion was found to the significantly $(t=3.17, \mathrm{P}<0.05)$ greater in the 206 un-supported condition. 
The aim of the current investigation was to examine the kinetics and kinematics of supported and un-supported landings of the rugby union line out. To the authors knowledge this

211 research represents the first to examine the biomechanics of lineout jumpers during different

212 conditions. The current investigation may give important information to coaches, clinicians

213 and officials regarding the appropriate implementation of the lineout.

215 The first key observation from the current investigation is that instantaneous load rate was

216 significantly larger in the un-supported condition in relation to the supported jumps. This

217 observation may have important implications as there is believed to be a strong association

218 between the magnitude of repeated impact loading and the aetiology of chronic lower limb

219 injuries (Whittle, 1999). Therefore, this investigation suggests that in un-supported conditions jumpers are at increased risk from injury in relation to being supported until they reach the ground.

The current investigation importantly showed that the vertical velocity of the jumpers at foot contact was significantly larger in the un-supported condition. It is proposed that this change vertical velocity relates to the vertical (upwards) forces applied to the jumper by the lifters in the supported line-out condition. This provided resistance to the constant acceleration caused by gravity and thus reduced the velocity of the jumper at the instance of foot contact. It is likely that the increased vertical velocity at the point of foot contact is the mechanism responsible for the larger instantaneous rate of loading that was observed during the unsupported lineouts. The rate of loading is proportional to the change in momentum of the body during landing (Whittle, 1999), therefore an increased vertical velocity of the body at 
the instance of foot contact will mediate a proportional change in the vertical loading rate experienced by the body (Whittle, 1999).

In addition, the findings from the current investigation confirmed that significant changes in sagittal plane kinematics at all of the lower extremity joints were evident between lifting conditions. Specifically it was shown that peak angles at the hip, knee and ankle and ranges of motion at the hip and knee joints were significantly larger in the un-supported condition. It is proposed that jumpers utilized these mechanical alterations to promote deceleration as a result of the increased vertical velocity observed in the un-supported condition (Derrick, 2004). These alterations in lower extremity biomechanics serve to reduce the bodies'

242 effective mass, and are utilized extensively in sports movements in response to a perceived high impact situation in order to decrease the proportion of total body mass that is decelerated during the impact phase (Derrick, 2004).

Of further importance to the current investigation is that limb stiffness was shown to be significantly larger in the supported in relation to the un-supported condition. This was to be expected given the kinematic observations as limb stiffness is expressed as a function of limb deformation under a given load (Farley \& Morgenroth, 1999). It is proposed that this alteration in limb stiffness is a result of the changes in sagittal plane kinematics that were observed between the line-out conditions, which served to mediate increases in limb

252 deformation. It is alleged that limb stiffness during the absorption phase preconditions the muscle-tendon units to store elastic energy, which may improve power production during explosive movements (Kyrolainen et al., 2001). It is currently unknown what implications 
this may have for performance at the line-out; but it is nonetheless an avenue that future investigations may wish to explore.

258 A potential limitation to the current research is the laboratory based nature of the data

259 collection protocol. Although this was necessary in order to scientifically obtain synchronous

260 kinetic and kinematic data in a controlled manner, the ecological validity of the procedure

261 from a practical context was compromised. Furthermore, in the interest of generating an

262 impartial comparison between the two line-out conditions the current investigation simulated

263 an attacking line-out, during which there was no requirement to continue play after the

264 jumper had landed. This indicates that the variants of the line-out that are dictated by the state

265 of play and the position of the set-piece on the pitch were not accounted for. Future work may wish to concentrate on the different variants of the line-out in order to provide a more comprehensive representation of the biomechanics of jumper during the line-out.

In conclusion, although the biomechanics of the line-out have been examined previously (Sayers, 2005; Trewartha et al., 2008), there is currently no information regarding the mechanics of the jumper and therefore the effect of supported and un-supported conditions on injury risk has not been investigated. As such the current investigation adds to the current knowledge by generating a comprehensive evaluation of both kinetic and kinematic 274 parameters measured during supported and un-supported line-outs. The results from this 275 investigation indicate that both instantaneous loading rate and vertical velocity at foot contact were significantly larger in the un-supported condition, despite lower body kinematics in this condition being modified in favour of deceleration. The findings from the current investigation indicate that if the line-out jumper is un-supported by the lifters in returning to 

the ground then increased exposure to the mechanisms linked to injury are likely to be greater. Therefore, given the number of line-outs that are conducted per game it is recommended that this law be clarified to also specify supported lowering of the jumper at all levels of play.

\section{References}

1. Arampatzis, A., Brüggemann, G. P., \& Metzler, V. (1999). The effect of speed on leg stiffness and joint kinetics in human running. Journal of Biomechanics, 32, 13491353.

2. Bathgate, A., Best, J. P., Craig, G., \& Jamieson, M. (2002). A prospective study of injuries to elite Australian rugby union players. British Journal of Sports Medicine, 36, 265-269.

3. Blickhan, R. (1989). The spring-mass model for running and hopping. Journal of Biomechanics, 22, 1217-1227.

4. Brooks, J. H., \& Kemp, S. P. T. (2011). Injury-prevention priorities according to playing position in professional rugby union players. British Journal of Sports Medicine, 45, 765-775.

5. Cappozzo, A., Catani, F., Della Croce, U., \& Leardini, A. (1995). Position and orientation in space of bones during movement: anatomical frame definition and determination. Clinical Biomechanics, 10, 171-178.

6. Croft, H., Chong, A., \& Wilson, B. (2011). Virtual reality assessment of rugby lineout throw kinematics. Sports Technology, 4, 2-12.

7. Derrick, T.R. (2004). The effects of knee contact angle on impact forces and accelerations. Medicine and Science in Sports and Exercise, 36, 832-837. 
8. Farley, C. T., \& Morgenroth, D. C. (1999). Leg stiffness primarily depends on ankle stiffness during human hopping. Journal of Biomechanics, 32, 267-273.

9. Graydon, R., Fewtrell, D., Atkins, S., \& Sinclair, J. (2015). The test-retest reliability of different ankle joint center location techniques. The Foot and Ankle Online Journal, 8, 1-11.

10. IRB. (2004). The Laws of the Game of Rugby Union. $\begin{array}{llll}\text { Retrieved } \quad \text { 5th } & \text { April, } & \text { 2008, }\end{array}$ http://www.irb.com/mm/document/newsmedia/0/071026ctirbanalysisrwc2007report\% 5f3830.pd

12. Patton, M. S., Johnstone, A. J., \& Smith, F. W. (2006). A complex fracture of the talus following a rugby union line-out. Journal of Science and Medicine in Sport, 9, 185-189.

13. Sayers, M. (2005). A three-dimensional analysis of lineout throwing in rugby union. In Science and Football V: The Proceedings of the Fifth World Congress on Sports Science and Football (p. 99). Routledge.

14. Sayers, M. G. (2011). Kinematic analysis of line-out throwing in elite international rugby union. Journal of Sports Science \& Medicine, 10, 553-558.

15. Sinclair, J., Hobbs, S. J., Currigan, G., \& Taylor, P. J. (2013). A comparison of several barefoot inspired footwear models in relation to barefoot and conventional running footwear. Comparative Exercise Physiology, 9, 13-21.

16. Sinclair, J., Taylor, P. J., Currigan, G., \& Hobbs, S. J. (2014). The test-retest reliability of three different hip joint centre location techniques. Movement \& Sport Sciences, 31-39. 

center location techniques. Journal of Applied Biomechanics, 31, 117-121. lineout throwing. Journal of sports sciences, 26, 845-854. beneath the foot: a review. Gait \& Posture, 10, 264-275.

Tables

Table 1: Hip joint kinematics as a function of un-supported and supported conditions.

\begin{tabular}{|c|c|c|c|c|c|}
\hline & \multicolumn{2}{|c|}{ Un-supported } & \multicolumn{2}{|c|}{ Supported } & \multirow[t]{3}{*}{ P-value } \\
\hline & Mean & $S D$ & Mean & $S D$ & \\
\hline \multicolumn{5}{|c|}{ Sagittal plane ( + =flexion \& - = extension) } & \\
\hline Angle at foot contact $\left({ }^{\circ}\right)$ & 26.8 & 13.7 & 24.4 & 13.8 & 0.41 \\
\hline Angle at landing termination $\left({ }^{\circ}\right)$ & 76.7 & 20.8 & 45.8 & 18.6 & 0.0001 \\
\hline Peak flexion $\left({ }^{\circ}\right)$ & 76.9 & 20.7 & 46.3 & 18.3 & 0.0001 \\
\hline $\operatorname{ROM}\left({ }^{\circ}\right)$ & 49.9 & 14.0 & 21.5 & 10.0 & 0.000002 \\
\hline Relative ROM $\left(^{\circ}\right)$ & 50.1 & 14.0 & 21.9 & 9.9 & 0.000004 \\
\hline
\end{tabular}

Notes: Bold/ italic p-values denote statistical significance

337 Table 2: Knee joint kinematics as a function of un-supported and supported conditions.

\begin{tabular}{|c|c|c|c|c|c|}
\hline & \multicolumn{2}{|c|}{ Un-supported } & \multicolumn{2}{|c|}{ Supported } & \multirow{3}{*}{ P-value } \\
\hline & Mean & $S D$ & Mean & $S D$ & \\
\hline \multicolumn{5}{|l|}{ Sagittal plane ( + =flexion $\&$ - = extension) } & \\
\hline Angle at foot contact $\left({ }^{\circ}\right)$ & 18.7 & 5.1 & 15.0 & 6.6 & 0.36 \\
\hline Angle at landing termination $\left({ }^{\circ}\right)$ & 93.1 & 22.6 & 63.2 & 16.2 & 0.00004 \\
\hline Peak flexion $\left({ }^{\circ}\right)$ & 93.1 & 22.6 & 63.2 & 16.2 & 0.00004 \\
\hline $\operatorname{ROM}\left({ }^{\circ}\right)$ & 74.4 & 22.7 & 48.2 & 14.3 & 0.0001 \\
\hline Relative ROM $\left({ }^{\circ}\right)$ & 74.4 & 22.7 & 48.2 & 14.3 & 0.0002 \\
\hline
\end{tabular}

Notes: Bold/ italic p-values denote statistical significance

Table 3: Ankle joint kinematics as a function of un-supported and supported conditions.

\begin{tabular}{|c|c|c|c|c|c|}
\hline & \multicolumn{2}{|c|}{ Un-supported } & \multicolumn{2}{c|}{ Supported } & \multirow{2}{*}{ P-value } \\
\hline $\begin{array}{c}\text { Sagittal plane (+=dorsiflexion \& - } \\
\text { plantarflexion) }\end{array}$ & Mean & SD & Mean & SD & \\
\hline
\end{tabular}




\begin{tabular}{|c|c|c|c|c|c|} 
Angle at foot contact $\left({ }^{\circ}\right)$ & -34.4 & 8.3 & -36.8 & 7.3 & 0.11 \\
\hline Angle at landing termination $\left({ }^{\circ}\right)$ & 15.5 & 7.7 & 14.2 & 7.3 & 0.21 \\
\hline Peak dorsiflexion $\left(^{\circ}\right)$ & 20.8 & 8.5 & 17.6 & 7.1 & 0.01 \\
\hline ROM $\left(^{\circ}\right)$ & 49.9 & 8.2 & 50.9 & 6.3 & 0.48 \\
\hline Relative $\operatorname{ROM}\left({ }^{\circ}\right)$ & 55.2 & 6.9 & 54.3 & 5.8 & 0.47 \\
\hline
\end{tabular}

Notes: Bold/ italic p-values denote statistical significance 